\title{
Identifying the culprit lesion in tumor induced hypophosphatemia, the solution of a clinical enigma
}

\author{
Mathilde M. Bruins Slot-Steenks $\mathbb{1}^{1} \cdot$ Neveen A.T. Hamdy ${ }^{1}$ • \\ Michiel A.J. van de Sande ${ }^{2}$ Dennis Vriens ${ }^{3}$ - Arjen H.G. Cleven ${ }^{4}$. \\ Natasha M. Appelman-Dijkstra ${ }^{1}$
}

Received: 18 April 2016 / Accepted: 13 August 2016 / Published online: 5 October 2016

(C) The Author(s) 2016; This article is published with open access at Springerlink.com

\begin{abstract}
Tumor-induced osteomalacia is a rare acquired metabolic bone disorder characterized by isolated renal phosphate wasting due to abnormal tumor production of fibroblast growth factor 23 . We report the case of a 59 year old woman referred to our department with a long history of progressive diffuse muscle weakness and pain, generalized bone pains and multiple insufficiency fractures of heels, ankles and hips due to a hypophosphatemic osteomalacia. A fibroblast growth factor 23-producing phosphaturic mesenchymal tumor localized in the left quadriceps femoris muscle was identified 7 years after onset of symptoms. Excision of the tumor resulted in normalization of serum phosphate and fibroblast growth factor 23 levels and in complete resolution of the clinical picture with disappearance of all musculoskeletal symptoms. This case illustrates the diagnostic difficulties in establishing a diagnosis tumor-induced osteomalacia and in identifying the responsible tumor. Our case underscores the clinical need to investigate all patients with persistent musculoskeletal symptoms for hypophosphatemia. A systematic approach is of pivotal importance because early recognition and
\end{abstract}

Mathilde M. Bruins Slot-Steenks

bot@lumc.nl

1 Center for Bone Quality and Department of Medicine, Division of Endocrinology, Leiden University Medical Center, Albinusdreef 2, Leiden 2333 ZA, The Netherlands

2 Center for Bone Quality and Department of Orthopedic Surgery, Leiden University Medical Center, Albinusdreef 2, Leiden 2333 ZA, The Netherlands

3 Department of Radiology, Leiden University Medical Center, Albinusdreef 2, Leiden 2333 ZA, The Netherlands

4 Department of Pathology, Leiden University Medical Center, Albinusdreef 2, Leiden 2333 ZA, The Netherlands treatment of the metabolic abnormality can prevent deleterious effects of osteomalacia on the skeleton.

Keywords Tumor-induced osteomalacia (TIO) - Fibroblast growth factor $23(\mathrm{FGF} 23) \cdot$ Hypophosphatemia

\section{Introduction}

Tumor-induced osteomalacia (TIO) is a rare acquired metabolic bone disorder occurring as a result of isolated renal phosphate wasting due to abnormal tumor production of fibroblast growth factor 23 (FGF23). Lack of awareness of the clinical manifestations of the disorder often leads to diagnostic delay, failure of diagnosis or misdiagnosis. Once a diagnosis TIO is suspected it is also often difficult to find the responsible FGF23-secreting tumor, since these tumors are often small, slow growing, benign tumors of mesenchymal origin, which can occur almost anywhere in bone or soft tissue [1-4]. Since the source of the FGF23 production is often very hard to find careful follow-up is necessary. Regular restaging should be performed until the culprit lesion is detected [3, 5]. In this article we do not only discuss the intense long diagnostic workup and follow-up of a patient with TIO but we also show that when patients are carefully monitored this can be performed without complications, e.g., tertiary hyperparathyroidism due to phosphate suppletion or new fractures.

\section{Case description}

A 59-year-old woman with a longstanding history of epilepsy with complex partial seizures, for which she had been 


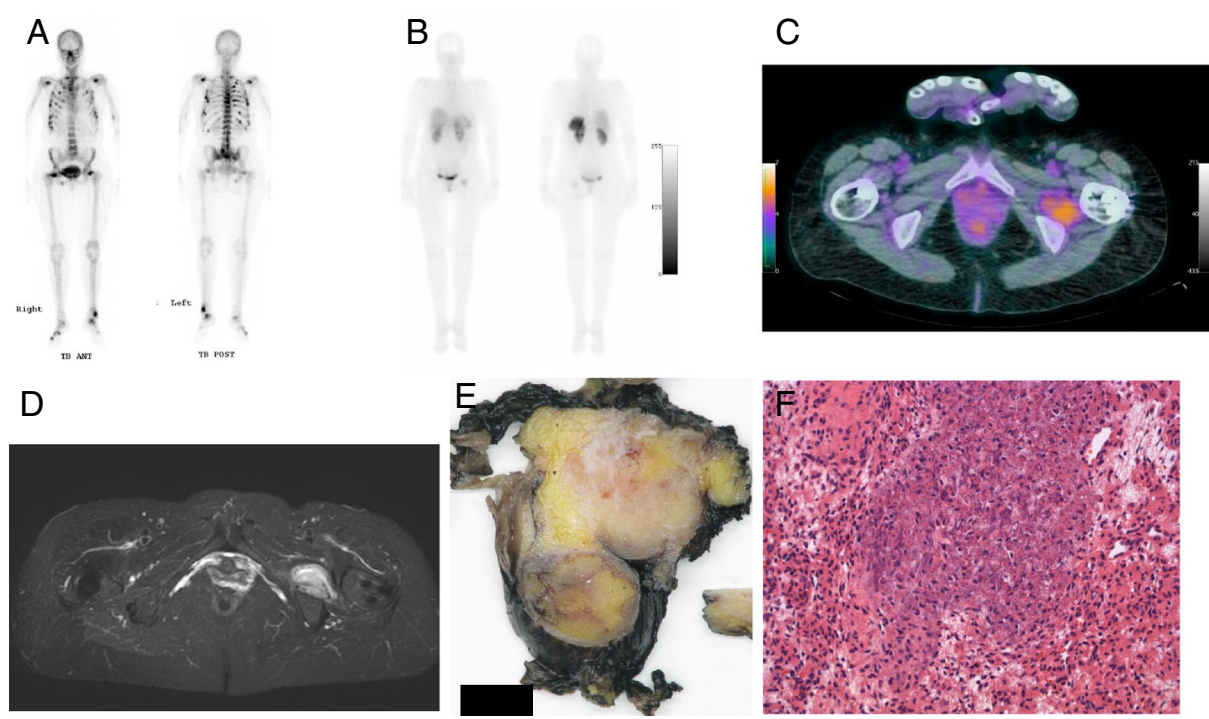

Fig. 1 a. Skeletal scintigraphy performed $4 \mathrm{~h}$ after injection of ${ }^{99 \mathrm{~m}} \mathrm{Tc}-$ hydroxydiphosphonate showing multiple focal areas of increased osteoblastic activity suggestive for microfractures together with general increased osteoblastic activity throughout the whole skeleton, suggestive for a metabolic bone disease. $\mathbf{b} .{ }^{111}$ Indium-pentetreotide planar total body scintigraphy (24 h after injection) showing pathological somatostatin receptor expression in the left inguinal region. c. Fusion transversal image of the ${ }^{\mathbf{1 8}}$ fluorodeoxyglucose positron emission tomography/computed tomography (FDG-PET/CT) showing a soft tissue lesion with moderately increased glucose metabolism (maximum standardized uptake value (SUVmax) $4.2 \mathrm{~g} / \mathrm{cm}^{3}$

using phenytoin for more than 20 years, was referred to our center with a 3-year history of progressive muscle weakness, associated with generalized bone and muscle pain, resulting in significant limitations in her daily activities. The referring physician's working diagnosis was osteoporosis secondary to long-term use of anti-convulsant therapy in the form of phenytoin. The diagnosis of osteoporosis was made on the basis of low bone mineral density measurements using dual-energy X-ray absorptiometry (lumbar $T$-score -2.2 standard deviations (SD), femoral neck $T$-score -3.0 $\mathrm{SD}$ ) and stress fractures of heels and ankles. The patient was also found to have a vitamin D deficiency, low serum calcium and phosphate concentration (respectively 2.13 and $0.45 \mathrm{mmol} / \mathrm{L}$ ) and elevated alkaline phosphatase (366 U/L). Treatment with phenytoin was discontinued. Supplementation of vitamin D in the form of colecalciferol (vitamin D3) and calcium carbonate was used in a dosage of $400 \mathrm{IE}$ and subsequently $500 \mathrm{mg} 3$ times per day. Subsequently intravenous treatment with bisphosphonates (pamidronate) was initiated before referral to our center. In view of persistence of complaints, a ${ }^{99 \mathrm{~m}} \mathrm{Tc}$-skeletal scintigraphy was performed, showing multifocal areas of increased radioactive isotope uptake, including at the femoral neck bilaterally, interpreted to be due to multiple insufficiency fractures (Fig. 1a). The patient was referred to our center for further investigation and management because persistence of symptoms and
47 min post-injection of $175 \mathrm{MBq}$ FDG) in the left inguinal region, situated in the $\mathrm{m}$. quadrates femoris. On the full-body images, no other FDG-avid lesions were detected. d. Transversal short tau inversion recovery (STIR) magnetic resonance image with an intermuscular located soft tissue mass inferior to the left hip joint. The lesion demonstrates inhomogeneous high signal intensity compared to muscle. e. Macroscopy shows a well circumscribed nodular soft tissue tumor (diameter $6 \mathrm{~cm}$ ). (black bar $=1 \mathrm{~cm}$ ). f. Microscopy shows a tumor composed of bland, spindle to stellate cells, surrounding a matrix of phosphaturic mesenchymal tumor that typically calcifies in a type of 'grungy' or flocculent fashion

hypophosphatemia and inadequate response of bone mineral density to bisphosphonate after an year of therapy.

At presentation in our center, the patients symptoms had further worsened with progressive proximal muscle pain and weakness and increasing bone pain. She was still using calcium/vitamin D supplementation. Laboratory investigation confirmed the presence of severe hypophosphatemia. In retrospect this was also the case at first presentation elsewhere. She had a normal renal and liver function, the serum calcium was still low in the presence of a normal parathormone, normal 25-hydroxyvitamin $\mathrm{D}$ and a low normal 1,25-dihydroxy vitamin D (Table 1). Bone turnover was elevated as suggested by high alkaline phosphatase. Conventional radiographs demonstrated Looser's zones in the femoral neck bilaterally. A diagnosis of hypophosphatemic osteomalacia was suspected. The 24-h urinary phosphate was $15 \mathrm{mmol} / 24 \mathrm{~h}$. The fractional tubular reabsorption of phosphate (TRP) was low 0.77 , calculated by the formula $\mathrm{TRP}=1-\{($ Urine phosphate $\times$ Serum creatinine $) /($ Serum phosphate $\times$ Urine creatinine) $\}$. Second morning-void urine and blood samples are needed taken at the same time without phosphate supplementation. When TRP is $\leq 0.86$ then the tubular maximum for phosphate corrected for glomerular filtration rate (TmP/GFR) can be calculated by the formula $\mathrm{TmP} / \mathrm{GFR}=\mathrm{TRP} \times$ serum phosphate, which was remarkable low $0.5 \mathrm{mmol} / \mathrm{L}$ (normal range $0.88-1.42$ 
Table 1 Laboratory investigation

\begin{tabular}{lllllll}
\hline Laboratory findings & $\begin{array}{l}\text { Initial } \\
\text { results }\end{array}$ & $\begin{array}{l}\text { At presentation } \\
\text { in our center }\end{array}$ & $\begin{array}{l}\text { 3 years } \\
\text { follow-up }\end{array}$ & $\begin{array}{l}\text { 4 years } \\
\text { follow-up }\end{array}$ & $\begin{array}{l}\text { Postoperative results } \\
\text { (5 days after resection) }\end{array}$ & $\begin{array}{l}\text { Reference } \\
\text { range }\end{array}$ \\
\hline phosphate $(\mathrm{mmol} / \mathrm{L})$ & 0.45 & 0.56 & 0.53 & 0.65 & 1.18 & $2.15-2.55$ \\
calcium $(\mathrm{mmol} / \mathrm{L})$ & 213 & 2.08 & 2.28 & 2.49 & 2.39 & $0.90-1.50$ \\
creatinine $(\mu \mathrm{mol} / \mathrm{L})$ & - & 57 & 62 & 85 & 65 & $49-90$ \\
alkaline phosphatase (U/L) & 366 & 271 & 115 & 148 & 102 & $0-98$ \\
PTH (pmol/L) & 7.0 & 7.8 & 4.4 & 1.6 & 5.4 & $1.5-8.0$ \\
vitamin D 25(OH) (nmol/L) & $\mathrm{nr}$ & 73 & 78 & 92 & 89 & $50-250$ \\
$1,25(\mathrm{OH}) 2$ vitamin D (pmol/L) & - & 58 & 80 & - & - & $40-140$ \\
C-FGF23 (*RU/L)/(**U/L) & - & $* 123$ & $* * 1370$ & $* * 2050$ & $* * 54$ & $* 0-120$ \\
& & & & & & $* * 0-125$ \\
\hline
\end{tabular}

$n r$ not retainable

mmol/L). TmP/GFR can also be determined using a nomogram [6]. The results were compatible with renal phosphate wasting. Considering the patient's age, normal phenotype and negative family history for bone and mineral disorders, a diagnosis of tumor-induced osteomalacia was considered. The C-terminal FGF-23 (Immutopics, San Clemente, CA, USA) was measured in ethylene-diaminetetraacetic acid (EDTA) plasma. The BioTek ELx50 (BioTek, Bad Friedrichshall, Germany) was used as an automatic washing machine. All analyses were performed according to manufacturer's protocol [7]. Serum C-terminal FGF23 was found to be above the upper limit of the normal laboratory reference of $123 \mathrm{RU} / \mathrm{mL}$ (normal value $<120$ $\mathrm{RU} / \mathrm{ml}$ ), inappropriately high in the presence of hypophosphatemia. An extensive search using different imaging tools including ${ }^{111}$ Indium-pentetreotide whole body single photon emission tomography (SPECT), computed tomography (CT) and magnetic resonance imaging (MRI), revealed no potential lesion as a source of FGF23 production. The patient was treated with active vitamin $\mathrm{D}$ metabolite (alfacalcidol 1dd1.5 $\mu \mathrm{g}$ ) and phosphate drink (3dd20 mmol), which partially increased serum phosphate level associated with improvement of symptoms. However, this was insufficient to control hypophosphatemia and associated symptoms in the long-term. There was a clear increase in C-terminal FGF23 (2050 RU/ml) over the following 4 years and it became progressively more difficult to control serum phosphate levels. Her clinical picture also significant worsened. She had difficulty in walking and surgical intervention was required to stabilize the severe insufficiency fractures of her hips. At that stage further radiological evaluation using ${ }^{111}$ Indium-pentetreotide scintigraphy and also a ${ }^{18}$ Fluorodeoxyglucose positron emission tomography/CT (FDG-PET/CT) was performed, both demonstrated a suspicious isolated lesion in the soft tissue at the left inguinal region (Fig. 1b, c). A MRI confirmed the presence of a small tumor in the left quadriceps femoris muscle (Fig. 1d), which was subsequently successfully resected. The resected lesion was a soft tissue tumor, with macroscopic features of a nodular tumor with a maximum diameter of $6 \mathrm{~cm}$ (Fig. 1e). Figure 1f shows the histology of the tumor composed of bland, spindle to stellate cells, surrounding a matrix of phosphaturic mesenchymal tumor that typically calcifies in a 'grungy' or flocculent fashion. Within 5 days after complete resection of the tumor, there was a rapid and complete normalization of serum phosphate and FGF23 levels associated with complete resolution of the clinical picture.

\section{Discussion}

Tumor-induced osteomalacia was first described by McCance in 1947 [8]. In 1959 Prader et al. were the first to recognize that the disease was the result of a tumor which secreted a 'rachitogenic' substance and that resection of the tumor resulted in resolution of the osteomalacia [9]. A growing recognition of the disease paralleled the identification of FGF23 as a phosphaturic agent in 2000 [10]. Despite unraveling the pathophysiology of TIO, there is still often delay in establishing the diagnosis because associated musculoskeletal symptoms are non-specific, the FGF23producing tumor is often very small and its localization very elusive. A delay in time between onset of symptoms and a further delay in localization and resection of the FGF23producing tumor may be associated with considerable morbidity because of progressive osteomalacia if the hypophosphatemia remains untreated or is more difficult to control if treated with active vitamin D metabolites as the tumor growth increases production of FGF23, resulting in increasing renal phosphate wasting. 
FGF23 is a hormone produced by osteocytes and osteoblasts. FGF23 acts primarily at the proximal renal tubule by inhibiting tubular phosphate reabsorption by reducing the expression of the sodium phosphate cotransporters NaPi-IIa and NaPi-IIc through activation of the receptor complex of fibroblast growth factor receptor $1 \mathrm{c}$ and its co-receptor klotho. FGF23 also inhibits the expression of the renal $1 \alpha$-hydroxylase enzyme and decreases thereby 1,25 -dihydroxy vitamin $\mathrm{D}$ production $[11,12]$. The pathognomonic features of excess circulating FGF23 are renal tubular wasting of phosphate and suppressed 1,25dihydroxy vitamin D levels which further impair intestinal absorption of phosphate worsening hypophosphatemia and resulting in impaired mineralization of bone.

A systematic approach to hypophosphatemia helps in the early recognition and treatment of TIO. Presenting symptoms of TIO are diffuse muscle and bone pain, initial proximal muscle weakness which becomes generalized and insufficiency fractures. A careful history taking and physical examination is important followed by laboratory investigations including calcium, phosphate, creatinine, parathyroid hormone (PTH) and vitamin D metabolites. All cases of hypophosphatemia should be further evaluated to establish whether low circulating levels of phosphate are due to renal phosphate wasting, decreased intestinal absorption of phosphate or to redistribution of phosphate from extracellular fluid into cells or into the bone matrix. Hypophosphatemia can also be caused by several medications. The underlying pathophysiological mechanism of medication-induced hypophosphatemia are as mentioned before or resulting from more than one mechanism. Our patient was known with prolonged therapy with anticonvulsants in the form of phenytoin. These drugs are inducers of the cytochrome P450 thereby causing increased vitamin $\mathrm{D}$ degradation. They also decrease calcium resorption in the gut [13]. In our case it was remarkable that the hypophosphatemia persisted after stopping phenytoin and vitamin D suppletion, so further evaluation was required. To determine if hypophosphatemia is the result of renal phosphate wasting it is necessary to calculate the TMP/GFR. Once renal phosphate wasting is determined the measurement of FGF23 is an important next step if parathyroid hormone concentration is in the normal range. FGF23 can be measured using two different assays, the intact FGF23 assay and the C-terminal FGF23 assay which detects intact FGF23 peptide and biologically inactive carboxyl terminal (C-terminal) fragments. Acquired hypophosphatemia due to renal phosphate wasting is most likely due to TIO, in which case excess FGF23 is most often produced by benign, small phosphaturic mesenchymal tumors [1-5, 14]. Malignant tumors and metastases are rare [5]. A benign phosphaturic mesenchymal tumor may occasionally progress to a high-grade osteosarcoma [15]. FGF23 secretion may also be observed in association with rare bone diseases such as neurofibromatosis, epidermal nevus syndrome and isolated fibrous dysplasia or in the setting of McCune-Albright [16-18]. Other possible causes of renal phosphate wasting with elevated FGF23 levels include inherited hypophosphatemic osteomalacia such as X-linked hypophosphatemic rickets, autosomal dominant hypophosphatemic rickets and autosomal recessive hypophosphatemic rickets. FGF23-independent causes of hypophoshataemia are alcohol abuse, drugs or toxins, renal tubular acidosis, Fanconi's syndrome or hereditary hypophosphatemic rickets with hypercalciuria.

The finding of hypophosphatemia in the absence of a family history for bone and mineral disorders, a normal phenotype and relatively recent onset of symptoms which may rapidly progress in an adult with previously documented normal serum phosphate suggest TIO, which may be confirmed by measuring FGF23 levels (Fig. 2). The definitive diagnosis of TIO is established by identification of the causative tumor and by resolution of the clinical picture following complete tumor resection. Complete surgical resection of the FGF23 secreting tumor is the only definitive therapy, which emphasizes the need for localizing FGF23-producing tumors. Localization of FGF23producing tumors can be challenging and a step-wise approach, including functional imaging, followed by anatomical imaging, and if necessary selective venous sampling may significantly improve success in tumor localization [4]. Tumors associated with osteomalacia variably express somatostatin receptors (SSTR1-5), allowing SSTR-based functional imaging by octreotide scintigraphy such as ${ }^{111}$ In-pentetreotide [19]. Because predominant overexpression of SSTR-2 subtype in tumors of TIO, ${ }^{68}$ Ga-octreotide PET/CT may have an important role in localization of the culprit lesion, and may potentially reduce significant delay in establishing the diagnosis of TIO [20]. FDG-PET/CT can confirm a lesion or identify a lesion that was not initially seen on octreotide scintigraphy $[4,20]$. FDG-PET/CT has been proven to be sensitive but not a specific method of identifying FGF23-producing tumors. PET/CT using 68-gallium is expected to be much more specific and informative for this kind of tumors but is often not available in many centers [5]. Anatomic imaging is used to confirm the localization of a tumor. When multiple suspicious lesions are identified selective venous sampling can increase diagnostic power by confirming the localization of the culprit lesion [21]. Furthermore establishing the diagnosis of an FGF23-producing phosphaturic mesenchymal tumor requires an adequate integration of the clinical picture in correlation with radiology and histology in a multidisciplinary setting, since radiology and histology show considerable overlap with other entities that need different treatment and harbor other clinical outcomes. 
Fig. 2 Suggested flowchart musculoskeletal symptoms and hypophosphatemia. TRP fractional tubular reabsorption of phosphate, TmP/GFR tubular maximum reabsorption of phosphate to glomerular filtration rate, $N$ normal, $P T H$ parathyroid hormone, Prim. $H P T$ primary hyperparathyroidism, Sec. $H P T$ secondary hyperparathyroidism, $F G F 23$ fibroblast growth factor 23, $F H$ family history, $H H R H$ hereditary hypophosphatemic rickets with hypercalciuria, $X L H$ $\mathrm{X}$-linked hypophosphatemic rickets, $A D H R$ autosomal dominant hypophosphatemic rickets, ARHR autosomal recessive hypophosphatemic rickets, $T I O$ tumor-induced osteomalacia, $F D$ Fibrous dysplasia, Mc Alb McCune Albright syndrome

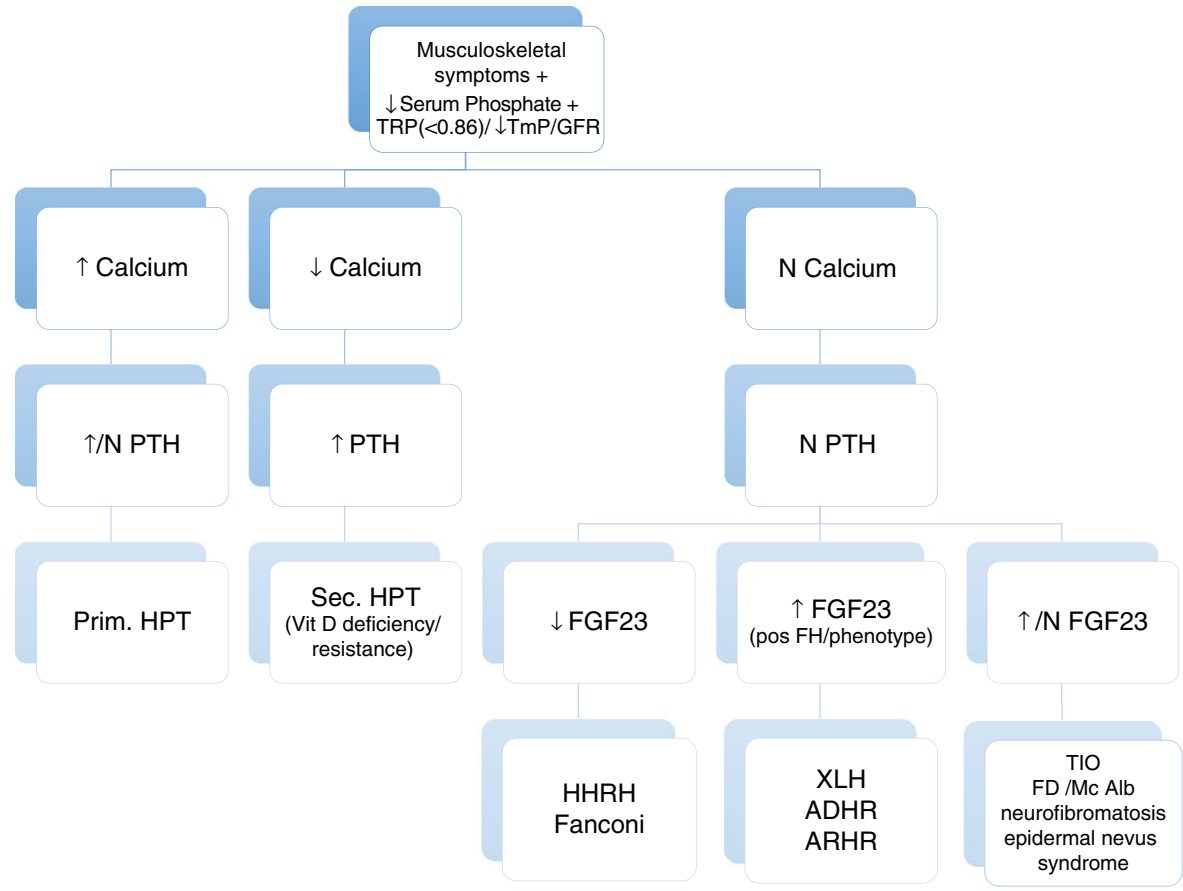

Since identifying the source of FGF23 overproduction may be challenging and often inconclusive in patients with TIO, medical treatment should be initiated. Phosphate treatment alone will not be sufficient and can be counterproductive since oral phosphate stimulates PTH secretion and is poorly absorbed due to impaired 1,25-dihydroxy vitamin D hydroxylation. Furthermore, the compliance of oral phosphate is rather low since this is often not well tolerated. Therefore treatment with active vitamin D metabolites is necessary since FGF23 not only increases urinary phosphate loss but inhibits the expression of $1 \alpha$-hydroxylase as well, leading to a decreased 25-hydroxyvitamin D to 1,25-dihydroxy vitamin $\mathrm{D}$ hydroxylation. Intestinal absorption of dietary phosphate will be impaired since the expression of type $2 \mathrm{~b}$ sodium phosphate co-transporter is downregulated due to the lack of 1,25-dihydroxy vitamin $\mathrm{D}$. Furthermore the decrease in 1,25-dihydroxy vitamin D stimulates the synthesis and secretion of PTH by the parathyroid glands thereby furthermore inducing urinary phosphate loss. By supplementation of active vitamin D metabolites you increase the intestinal absorption of phosphate and suppress PTH production and should therefore be the drug of choice. In cases the hypophosphatemia is severe oral phosphate can be added with close monitoring of the PTH levels and urinary calcium excretion since this will increase urinary calcium excretion which can lead to nephrocalcinosis and nephrolithiasis. Therapy should be continued for as long as the tumor is not identified or resected. Patients should also be closely monitored for complications of the hypophosphatemia, because longstanding hypophosphatemia can lead to progression of osteomalacia and associated increased risk of fractures. Adenosine triphosphate (ATP) depletion in hypophosphatemia can also effect the cardiopulmonary system by impaired myocardial and diaphragmatic contractility and effect the hematopoietic system leading to hemolysis and leukocyte dysfunction, these complications are rare [22]. The search for the source of FGF23 should also continue using state of the art imaging techniques.

Surgical intervention is curative when a lesion is identified and fully resected. Radical excision is important to avoid a recurrence. After successful tumor resection, the serum level of FGF23 decreases to a normal level within a few hours to days depending on the magnitude of the initial elevation (half-life of FGF23 is approximately 46-58 min) [23]. Improvement of clinical symptoms rapidly follows with subsequent complete remineralization of the skeleton [19]. Late recurrence due to local recurrence or distant metastasis is very uncommon and occurs in approximately less than $5 \%$ of patients with TIO [2, 3]. Long-term followup is recommended, FGF23 can be used as a tumor marker for early detection of recurrences.

\section{Conclusion}

Tumor-induced osteomalacia represent a significant diagnostic challenge and should be considered in the differential diagnosis of all patients with musculoskeletal symptoms associated with hypophosphatemia. Association of hypophosphatemia, renal phosphate wasting (low TRP/low TmP/ GFR), elevated FGF23 levels and paradoxically low or 
normal 1,25-dihydroxy vitamin D suggests the diagnosis of TIO after exclusion of the hereditary hypophosphatemic osteomalacia by adult onset of hypophosphatemia in the absence of family history and characteristic phenotype. Awareness of this acquired metabolic abnormality, a systematic approach and evaluation of a hypophosphatemia in a multidisciplinary setting are of pivotal importance because early recognition and treatment can prevent progression of osteomalacia and associated increased risk of fractures. Patients should be prescribed active metabolites of vitamin $\mathrm{D}$ with a few requiring additional phosphate supplementation. After the FGF23-producing tumor is located and fully resected, long-term follow-up is advocated for early diagnosis of a potential recurrence.

\section{Compliance with ethical standards}

Conflict of interest The authors declare that they have no conflict of interests.

Open Access This article is distributed under the terms of the Creative Commons Attribution 4.0 International License (http:// creativecommons.org/licenses/by/4.0/), which permits unrestricted use, distribution, and reproduction in any medium, provided you give appropriate credit to the original author(s) and the source, provide a link to the Creative Commons license, and indicate if changes were made.

\section{References}

1. N. Weidner, D.S. Cruz, Phosphaturic mesenchymal tumors a polymorphous group causing osteomalacie or rickets. Cancer 59, 1442-1454 (1987)

2. A.L. Folpe, J.C. Fanburg-Smith, S.D. Billings, M. Bisceglia, F. Bertoni, J.Y. Cho et al., Most osteomalacia-associated mesenchymal tumors are a single histopathologic entity: an analysis of 32 cases and a comprehensive review of the literature. Am J Surg Pathol. 28(1), 1-30 (2004)

3. Y. Jiang, W.B. Xia, X.P. Xing, B.C. Silva, M. Li, O. Wang et al., Tumor-induced osteomalacia: an important cause of adult-onset hypophosphatemic osteomalacia in China: Report of 39 cases and review of the literature. J Bone Miner Res. 27(9), 1967-1975 (2012)

4. W.H. Chong, P. Andreopoulou, C.C. Chen, J. Reynolds, L. Guthrie, M. Kelly et al., Tumor localization and biochemical response to cure in tumor-induced osteomalacia. J Bone Miner Res. 28(6), 1386-1398 (2013)

5. W.H. Chong, A.A. Molinolo, C.C. Chen, M.T. Collins, Tumorinduced osteomalacia. Endocr Relat Cancer 18(3), R53-R77 (2011)

6. O.L. Bijvoet, Relation of plasma phosphate concentration to renal tubular reabsorption of phosphate. Clin Sci. 37(1), 23-36 (1969)
7. A.C. Heijboer, M. Levitus, M.G. Vervloet, P. Lips, P.M. ter Wee, H.M. Dijstelbloem et al., Determination of fibroblast growth factor 23. Ann Clin Biochem. 46, 338-340 (2009)

8. R.A. McCance, Osteomalacia with Looser's nodes (Milkman's syndrome) due to a raised resistance to vitamin $\mathrm{D}$ acquired about the age of 15 years. Q J Med. 16(1), 33-46 (1947)

9. A. Prader, R. Illig, E. Uehlinger, G. Stalder, Rickets following bone tumor. Helv Paediatr Acta. 14, 554-565 (1959)

10. ADHR Consortium, Autosomal dominant hypophosphataemic rickets is associated with mutations in FGF23. Nat Genet. 26(3), 345-348 (2000)

11. F. Perwad, M.Y. Zhang, H.S. Tenenhouse, A.A. Portale, Fibroblast growth factor 23 impairs phosphorus and vitamin D metabolism in vivo and suppresses 25-hydroxyvitamin D-1alphahydroxylase expression in vitro. Am J Physiol Renal Physiol. 293 (5), F1577-F1583 (2007)

12. X. Yan, H. Yokote, X. Jing, L. Yao, T. Sawada, Y. Zhang et al., Fibroblast growth factor 23 reduces expression of type IIa Na+/Pi co-transporter by signaling through a receptor functionally distinct from the known FGFRs in opossum kidney cells. Genes Cells. 10 (5), 489-502 (2005)

13. G. Liamis, H.J. Milionis, M. Elisaf, Medication-induced hypophosphatemia: a review. QJM. 103(7), 449-459 (2010)

14. N. Weidner, Review and update: oncogenic osteomalacia-rickets. Ultrastruct Pathol. 15(4-5), 317-333 (1991)

15. A. Ogose, T. Hotta, I. Emura, H. Hatano, Y. Inoue, H. Umezu et al., Recurrent malignant variant of phosphaturic mesenchymal tumor with oncogenic osteomalacia. Skeletal Radiol. 30(2), 99-103 (2001)

16. R. Ivker, S.D. Resnick, R.A. Skidmore, Hypophosphatemic vitamin D-resistant rickets, precocious puberty, and the epidermal nevus syndrome. Arch Dermatol. 133(12), 1557-1561 (1997)

17. K. Konishi, M. Nakamura, H. Yamakawa, H. Suzuki, T. Saruta, H. Hanaoka et al., Hypophosphatemic osteomalacia in von Recklinghausen neurofibromatosis. Am J Med Sci. 301(5), 322-328 (1991)

18. M. Riminucci, M.T. Collins, N.S. Fedarko, N. Cherman, A. Corsi, K.E. White et al., FGF-23 in fibrous dysplasia of bone and its relationship to renal phosphate wasting. J Clin Invest. 112(5), 683-692 (2003)

19. S.M. Jan de Beur, E.A. Streeten, A.C. Civelek, E.F. McCarthy, L. Uribe, S.J. Marx et al., Localisation of mesenchymal tumours by somatostatin receptor imaging. Lancet. 359(9308), 761-763 (2002)

20. R.J. Clifton-Bligh, M.S. Hofman, E. Duncan, I. Sim, D. Darnell, A. Clarkson et al., Improving diagnosis of tumor-induced osteomalacia with Gallium-68 DOTATATE PET/CT. J Clin Endocrinol Metab. 98(2), 687-694 (2013)

21. P. Andreopoulou, C.E. Dumitrescu, M.H. Kelly, B.A. Brillante, C. M. Cutler Peck, F.M. Wodajo et al., Selective venous catheterization for the localization of phosphaturic mesenchymal tumors. J Bone Miner Res. 26(6), 1295-1302 (2011)

22. J. Amanzadeh, R.F. Reilly, Hypophosphatemia: an evidencebased approach to its clinical consequences and management. Nat Clin Pract Nephrol. 2(3), 136-148 (2006)

23. A. Khosravi, C.M. Cutler, M.H. Kelly, R. Chang, R.E. Royal, R. M. Sherry et al., Determination of the elimination half-life of fibroblast growth factor-23. J Clin Endocrinol Metab. 92(6), 2374-2377 (2007) 